\title{
ARTICLE
}

Infectious medicine, virology

\section{COVID-19 in persons with haematological cancers}

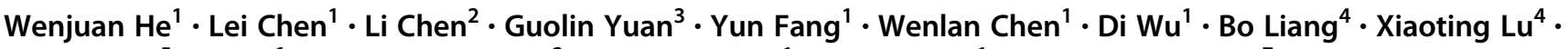 \\ Yanling $\mathrm{Ma}^{5} \cdot$ Lei Li $^{6} \cdot$ Hongxiang Wang ${ }^{2} \cdot$ Zhichao Chen $^{1} \cdot$ Qiubai Li $\mathbb{I}^{1} \cdot$ Robert Peter Gale $^{7}$
}

Received: 1 April 2020 / Revised: 3 April 2020 / Accepted: 6 April 2020 / Published online: 24 April 2020

(c) The Author(s), under exclusive licence to Springer Nature Limited 2020

\begin{abstract}
Infection with SARS-CoV-2, the cause of coronavirus infectious disease-19 (COVID-19), has caused a pandemic with $>850,000$ cases worldwide and increasing. Several studies report outcomes of COVID-19 in predominately well persons. There are also some data on COVID-19 in persons with predominately solid cancer but controversy whether these persons have the same outcomes. We conducted a cohort study at two centres in Wuhan, China, of 128 hospitalised subjects with haematological cancers, 13 (10\%) of whom developed COVID-19. We also studied 226 health care providers, 16 of whom developed COVID-19 and 11 of whom were hospitalised. Co-variates were compared with the 115 subjects with haematological cancers without COVID-19 and with 11 hospitalised health care providers with COVID-19. There were no significant differences in baseline co-variates between subjects with haematological cancers developing or not developing COVID-19. Case rates for COVID-19 in hospitalised subjects with haematological cancers was 10\% (95\% Confidence Interval $[\mathrm{CI}], 6,17 \%)$ compared with $7 \%(4,12 \% ; P=0.322)$ in health care providers. However, the 13 subjects with haematological cancers had more severe COVID-19 and more deaths compared with hospitalised health care providers with COVID-19. Case fatality rates were $62 \%(32,85 \%)$ and $0(0,32 \% ; P=0.002)$. Hospitalised persons with haematological cancers have a similar case rate of COVID-19 compared with normal health care providers but have more severe disease and a higher case fatality rate. Because we were unable to identify specific risk factors for COVID-19 in hospitalised persons with haematological cancers, we suggest increased surveillance and possible protective isolation.
\end{abstract}

These authors contributed equally: Wenjuan He, Lei Chen, Li Chen, Guolin Yuan, Yun Fang

Supplementary information The online version of this article (https:// doi.org/10.1038/s41375-020-0836-7) contains supplementary material, which is available to authorised users.

Hongxiang Wang

whitely1972@sina.com

$\triangle$ Zhichao Chen

chenzhichao@hust.edu.cn

$\triangle$ Qiubai Li

qiubaili@hust.edu.cn

1 Institute of Haematology, Union Hospital, Tongji Medical College, Huazhong University of Science and Technology, Wuhan 430022, China

2 Department of Haematology, Wuhan Central Hospital, Tongji Medical College, Huazhong University of Science and Technology, Wuhan, China

\section{Introduction}

There have been several epidemic and clinical studies of community-acquired SARS-CoV-2-infection and coronavirus infectious disease-19 (COVID-19) [1-13]. There are also several studies of COVID-19 in persons with solid cancers, including those who are hospitalised for reasons other than COVID-19; whether these persons have a higher risk of developing COVID-19 after SARS-CoV-2-infection

3 Department of Haematology, Xiangyang Central Hospital, The Affiliated Hospital of Hubei University of Arts and Science, Xiangyang 441021, China

4 Department of Radiology, Union Hospital, Tongji Medical College, Huazhong University of Science and Technology, Wuhan 430022, China

5 Department of Respiratory, Union Hospital, Tongji Medical College, Huazhong University of Science and Technology, Wuhan 430022, China

6 Wuhan Jin-Yin-Tan Hospital, Wuhan, China

7 Centre for Haematology Research, Department of Immunology and Inflammation, Imperial College London, London, UK 
is controversial as is whether they have a worse prognosis [14-18].

There are few data on risk of developing COVID-19 in hospitalised persons with haematological cancers. Many, if not most persons with haematological cancers receive anticancer drugs with suppress bone marrow function or have cancers of the immune system and are at substantial risk of community- and hospital-acquired infections [19-21].

We analysed data of 128 hospitalised subjects with haematological cancers in Wuhan, China, 13 of whom developed COVID-19. Data were compared with 115 hospitalised subjects with haematological cancers without COVID-19 and with 11 health care providers with COVID19. We show hospitalised subjects with haematological cancers who develop COVID-19 have more severe disease, and a substantially higher risk of death compared with health care providers with COVID-19. Hospitalised persons with haematological cancers at great risk to develop COVID-19 cannot be accurately prospectively identified. As such, these persons should receive heighten surveillance and protective isolation should be considered.

\section{Methods}

\section{Study design}

Beginning 23 January 2020, when Wuhan was locked down at the onset of the outbreak, people without COVID-19 were not admitted to our hospitals to avoid person-to-person transmission. We analysed data as of 14 February 2020. Centres included all hospitalised persons at two main haematology centres in Wuhan (Union Hospital and Wuhan Central Hospital, Tongji Medical College, Huazhong University of Science and Technology). Haematological cancers were classified according to the 2016 WHO classification.

In Wuhan, quantitative real-time polymerase chain reaction (qRT-PCR) testing for the diagnosis of SARSCoV-2-infection was not available at our hospitals before 27 January 272020 and not used for routine screening before mid-February. Consequently, diagnosis of COVID-19 was based initially on lung computed tomography (CT) scans. $[12,22]$. All hospitalised persons with haematological cancers and health care providers regardless of whether the symptoms consistent with COVID-19 had a screening lung CT scan. If the screening lung CT was normal it was repeated when/if the person developed signs or symptoms consistent with COVID-19. All cases of COVID-19 by lung CT had a diagnostic assay for SARS-CoV-2-infection by qRT-PCR of nasal and oropharyngeal swab specimens (Shanghai Bio-Germ Medical Technology Co Ltd) according to the WHO interim guidance [23] and the Novel
Coronavirus Pneumonia Diagnosis and Treatment Program of the National Health Commission of China [22].

Onset time of COVID-19 in persons with haematological cancers was based on comprehensive analyses of signs and symptoms and/or a lung CT scan. Severity of COVID-19 was graded as follows [22]: (1) mild; mild clinical symptoms, no pneumonia on lung CT; (2) common: fever, cough and lung CT with pneumonia; (3) severe: respiratory distress (respiratory rate $>30 \mathrm{~min}^{-1}$, oxygen saturation $\left(\mathrm{O}_{2 \mathrm{Sat}}\right) \leq 93 \%$ at rest and/or ratio of arterial oxygen partial pressure to fractional inspired oxygen $\leq 300 \mathrm{mmHg}\left(\mathrm{P}_{\mathrm{aO} 2} /\right.$ $\mathrm{F}_{\mathrm{IO} 2}$ ); and (4) critical: aforementioned criteria of respiratory failure receiving mechanical ventilation, shock and/or organ failure other than lung and/or intensive care unit (ICU) hospitalisation. All the hospitalised subjects were transferred to isolation wards in the department of infectious disease when they were diagnosed with COVID-19. Hospitalised health care providers were used as a comparator cohort. The study was approved by the Ethics Committees of Wuhan Central Hospital (2020-007) and of Union Hospital (2020-0095). Written informed consent from subjects was waived by the Ethics Committees.

\section{Data collection}

Epidemiological, clinical and laboratory data, radiology reports, therapy details and outcomes on patients were obtained by accessing standardised forms from electronic medical records. Data on non-hospitalised health care providers was obtained using standardised forms. Data collection forms were reviewed independently by two researchers; a third researcher adjudicated discordances.

\section{Outcomes}

Outcomes were defined as follows: (1) death; (2) cure: two successive negative qRT-PCR tests $>24 \mathrm{~h}$ apart and asymptomatic; (3) improved: improvement in signs and symptoms and laboratory parameters and no progression on lung CT; (4) progressing: increase in symptoms and/or progression of lung CT findings and (5) stable: improved nor progressing.

\section{Statistical analysis}

Continuous variables were expressed as mean (SD) for normally distributed data or as the median (IQR) for abnormally distributed data. Categorical variables were frequency rates and percentages. Means were compared using independent group $t$-test and Mann-Whitney test for normally and abnormally distributed data. Proportions for categorical variables were compared by $\chi^{2}$ tests. $\mathrm{R}$ version 3.5.2 was used for statistical analyses. For unadjusted 
comparisons, a two-sided alpha of $<0.05$ was considered significant. Analyses were not adjusted for multiple comparisons. Consequently, findings should be interpreted as exploratory and descriptive.

\section{Results}

\section{Baseline characteristics of subjects with haematological cancer and COVID-19}

We enroled 224 health care providers and 128 hospitalised subjects with haematological cancers (Supplementary Tables 1 and 2). Most hospitalised subjects had acute myeloid leukaemia (AML; $N=50$ ), acute lymphoblastic leukaemia (ALL; $N=26$ ), non-Hodgkin lymphoma (NHL; $N=18)$, plasma cell myeloma (PCM; $N=19$ ) or myelodysplastic syndrome (MDS; $N=8$ ).

Thirteen of the one hundred and twenty-eight hospitalised subjects with a haematological cancer (10\%; 95\% Confidence Interval (CI), 6, 17\%) developed COVID-19 including four with AML, five with ALL, three with PCM and one with MDS (Table 1). There was no case of COVID19 amongst 18 subjects with NHL. There was no correlation between type of haematological cancer and likelihood of developing COVID-19 when analysed by leukaemia versus lymphoma or myeloid versus lymphoid cancer. Seven subjects in the COVID-19 cohort were male versus 65 in the non-COVID-19 cohort $(P>0.99)$. Median age of subjects with and without COVID-19 was 35 year (range, 23-53 years) and 49 year (range, $33-59$ years; $P=0.082$ ). Cardioand cerebrovascular diseases were the most common comorbidities with similar prevalence (24\%). Overall, 122 of the 128 subjects with haematological cancers (95\%) received prior anti-cancer treatment including chemotherapy $(N=76)$, molecular targeted therapy $(N=9)$, immune therapy $(N=23)$ or a proteasome inhibitor $(N=9)$ before $(N=75)$ or after $(N=110)$ hospitalisation. Median interval from the end of the last cycle of anti-cancer therapy to diagnosis of COVID-19 was 9 days (range, 7-19 days) in the six subjects who had previously received drugs damaging the bone marrow. Thirty-two $(25 \%)$ received $\geq 4$ cycles of anti-cancer therapy previously. There was no difference in numbers of prior anti-cancer cycles $(P=0.783)$ or disease state (remission or not) of haematological cancers $(P=0.670)$ between the COVID-19 and non-COVID-19 haematological cancer cohorts. Ten of thirteen subjects with COVID-19 and one hundred of one hundred and fifteen (87\%) subjects without COVID-19 received anti-cancer treatment after admission and before the symptom onset of COVID-19.

Overall, 16 of 224 health care providers $(7.1 \%$ [4, 12\%] developed COVID-19. This case rate is like the case rate in hospitalised subjects with haematological cancers $(P=$ 0.322). Three of the thirteen hospitalised haematological cancer subjects (subjects 6, 13 and 14) were initially diagnosed by a screening lung CT and 10 (subjects 1, 2, 4, 5 and $7-12$ ) by a lung CT done after developing symptoms. One of sixteen health care providers (patient 16) was diagnosed by a screening lung $\mathrm{CT}$ and the remainder (subjects 17-31) by a lung CT done after developing symptoms. The distribution for the 11 hospitalised health care providers was 1 (subject 16) diagnosed by a screening lung CT and 10 (subjects $21-25$ and 27-31) by a lung CT after symptoms.

\section{Clinical co-variates and outcomes of subjects with haematological cancer and COVID-19}

The 11 hospitalised health care providers were used as a comparator cohort. Nine were female. Median age was 32 years (range, 29-36 years). Nine were nurses, one, a physician and one, a health care attendant. (Supplementary Tables 1, 3 and 6). The 13 hospitalised subjects with haematological cancer and COVID-19 and the 11 hospitalised health care providers with COVID-19 had similar sex and age distributions (Table 2). Twelve haematological cancer subjects became febrile compared with four health care providers $(P=0.008)$, cough, twelve compared with four $(P=0.008)$ and dyspnoea, ten compared with three $(P=0.043)$. Lung CT scans of subjects with COVID-19 showed typical changes including patchy shadows, ground-glass opacity, air space consolidation or complete opacity (whiteout) [16] but were similar between the cohorts (Fig. 1). At the start of the disease, subjects with haematological cancer and COVID-19 had significantly higher levels of C-reactive protein (CRP) and procalcitonin, lower haemoglobin, lymphocyte and platelet concentrations but similar levels of lactate dehydrogenase (LDH), aspartate aminotransferase (AST), alanine aminotransferase, bilirubin, creatinine and blood urea nitrogen, compared with the cohort without haematological cancer. Some or all of these differences may be related to therapy of the haematological cancers rather than COVID-19.

After symptom onset of COVID-19 subjects with haematological cancer had significantly decreased haemoglobin, lymphocyte, lymphocyte subset, platelet and concentrations and higher concentrations of D-dimer $(P=0.001)$, AST, LDH, CRP, procalcitonin and ferritin compared with health care providers with COVID-19 but not different concentrations of cytokines including interleukins- $6,-2,-4$ and -10 , tumour necrosis factor-alpha and interferon-gamma (Supplementary Table 4). Subjects with haematological cancer and COVID-19 had more co-infections including bacteria $(N=$ $11)$, fungi $(N=9)$ and other viruses $(N=7)$. Only three health care providers with COVID-19 had a bacterial co-infection. Subjects with haematological cancer and COVID-19 had more complications including six with 
Table 1 Baseline co-variates of subjects with haematological cancer and COVID-19.

\begin{tabular}{|c|c|c|c|c|}
\hline & $\begin{array}{l}\text { Total, } \\
n=128\end{array}$ & $\begin{array}{l}\text { COVID-19 } \\
N=13\end{array}$ & $\begin{array}{l}\text { Non-COVID-19 } \\
N=115\end{array}$ & $P$ value \\
\hline Age, median (IQR), years & $49(31,59)$ & $35(23,53)$ & $49(33,59)$ & 0.082 \\
\hline Male sex & $72(565)$ & 7 & $65(57 \%)$ & $>0.999$ \\
\hline \multicolumn{5}{|l|}{ Co-morbidities } \\
\hline ASCVD & $31(245)$ & $3(23)$ & $28(24 \%)$ & $>0.999$ \\
\hline Diabetes & 8 & 0 & 8 & 0.706 \\
\hline Digestive system disease & 7 & 0 & 7 & 0.830 \\
\hline Hepatitis B & 7 & 0 & 7 & 0.830 \\
\hline Other cancer & 2 & 0 & 2 & $>0.999$ \\
\hline \multicolumn{5}{|l|}{ Exposure } \\
\hline $\begin{array}{l}\text { Huanan Seafood Wholesale Market } \\
\text { exposure }\end{array}$ & 1 & 0 & 1 & $<0.001$ \\
\hline Contact with suspected persons & 0 & 0 & 0 & \\
\hline $\begin{array}{l}\text { Contact with clinically diagnosed } \\
\text { persons }\end{array}$ & 2 & 2 & 0 & \\
\hline Contact with confirmed persons & 1 & 1 & 0 & \\
\hline \multicolumn{5}{|l|}{ Cancer } \\
\hline Acute myeloid leukaemia & $50(39 \%)$ & 4 & $46(40 \%)$ & 0.729 \\
\hline Acute lymphoblastic leukaemia & $26(20 \%)$ & 5 & $21(18 \%)$ & 0.176 \\
\hline Plasma cell myeloma & $19(15 \%)$ & 3 & $16(14 \%)$ & 0.639 \\
\hline Myelodysplastic syndromes & 8 & 1 & 7 & $>0.999$ \\
\hline Non-Hodgkin lymphoma & $18(14 \%)$ & 0 & $18(16 \%)$ & 0.264 \\
\hline \multicolumn{5}{|l|}{ Therapy } \\
\hline Chemotherapy & $76(59)$ & 6 & $70(61 \%)$ & 0.468 \\
\hline Allotransplant & 10 & 3 & 7 & 0.106 \\
\hline Targeted drug & 9 & 1 & 8 & $>0.999$ \\
\hline Immune suppression & $23(18 \%)$ & 2 & $21(18 \%)$ & $>0.999$ \\
\hline Proteasome inhibitor & 9 & 2 & 7 & 0.502 \\
\hline \multicolumn{5}{|l|}{ Prior anti-cancer therapy (cycles) } \\
\hline 0 & $50(39 \%)$ & 7 & $43(38 \%)$ & \\
\hline 1 & $19(15 \%)$ & 1 & $18(16 \%)$ & 0.783 \\
\hline 2 & 7 & 1 & 6 & \\
\hline 3 & $17(13 \%)$ & 1 & $16(14 \%)$ & \\
\hline$\geq 4$ & $32(25 \%)$ & 3 & $29(25 \%)$ & \\
\hline $\begin{array}{l}\text { Anti-cancer treatment after } \\
\text { admission }\end{array}$ & $110(86 \%)$ & 10 & $100(87 \%)$ & 0.572 \\
\hline \multicolumn{5}{|l|}{ Disease state } \\
\hline Newly diagnosed & 14 & 1 & 13 & 0.668 \\
\hline Unevaluated & $28(22 \%)$ & 3 & $25(22 \%)$ & \\
\hline CR & $35(27 \%)$ & 3 & $32(28 \%)$ & \\
\hline Non-CR & $32(25 \%)$ & 3 & $29(25 \%)$ & \\
\hline Progression & 7 & 0 & 7 & \\
\hline Relapse & 12 & 3 & 9 & \\
\hline
\end{tabular}

Data are $N(\%)$ unless specified otherwise.

$A S C V D$ cardio- and cerebro-vascular disease, $C R$ complete remission.

aHaematological cancer subjects with and without COVID-19 (COVID-19/non-COVID-19). 
Table 2 Clinical and laboratory co-variates, therapy and outcomes of subjects with haematological cancer and COVID-19.

\begin{tabular}{|c|c|c|c|}
\hline & Haematological cancer, $N=13$ & Health care providers ${ }^{\mathrm{a}}, N=11$ & $P$ value \\
\hline Age, median (IQR), years & $35(23,53)$ & $32(28,36)$ & 0.794 \\
\hline Male sex & & & 0.227 \\
\hline Male & 7 & 2 & \\
\hline \multicolumn{4}{|l|}{ Signs and symptoms at onset } \\
\hline Fever & 12 & 4 & 0.008 \\
\hline Cough & 12 & 4 & 0.008 \\
\hline Dyspnoea & 10 & 3 & 0.043 \\
\hline Muscle ache & 1 & 0 & $>0.999$ \\
\hline Headache & 1 & 0 & $>0.999$ \\
\hline Diarrhoea & 3 & 2 & $>0.999$ \\
\hline Fever, cough and shortness of breath & 8 & 3 & 0.205 \\
\hline \multicolumn{4}{|l|}{ Lung CT scan $(N=11)^{\mathrm{b}}$} \\
\hline Patchy shadows & 2 & 2 & $>0.999$ \\
\hline Ground-glass opacity & 8 & 9 & \\
\hline Air space consolidation/complete opacity & 1 & 0 & \\
\hline \multicolumn{4}{|c|}{ Laboratory findings (normal range), median $(\mathrm{IQR})^{\mathrm{c}}$} \\
\hline $\mathrm{WBC} \times 10 \mathrm{E}+9 / \mathrm{L}(3.5-9.5)$ & $3.9(1.0,9.4)$ & $6.1(5.3, .6)$ & 0.470 \\
\hline Neutrophils, $\times 10 \mathrm{E}+9 / \mathrm{L}(1.8-6.3)$ & $1.9(0.4,7.3)$ & $4.1(2.9, .5)$ & 0.337 \\
\hline Lymphocytes $\times 10 \mathrm{E}+9 / \mathrm{L}(1.1-3.2)$ & $0.5(0.3,0.9)$ & $1.5(1.2, .1))$ & 0.012 \\
\hline Platelets, ×10E + 9/L (125-350) & $67(24,80)$ & $247(210,274)$ & $<0.001$ \\
\hline $\begin{array}{l}\text { Haemoglobin, g/L (male, 130-175; } \\
\text { female, 115-150) }\end{array}$ & $69(62,89)$ & $132(125,134)$ & $<0.001$ \\
\hline Prothrombin time, s (11-16) & $16(14,17)$ & $13(12,13)$ & 0.105 \\
\hline $\begin{array}{l}\text { Activated partial thromboplastin time, s } \\
(28.0-43.5)\end{array}$ & $41.8(34.2,47.4)$ & $28.8(26.9,30.6$ & 0.0148 \\
\hline D-dimer, mg/L $(<0.5)$ & $0.8(0.6,1.3)$ & $0.5(0.4,0.7)$ & 0.476 \\
\hline Alanine aminotransferase, U/L (9-52) & $17(12,27)$ & $21(17,26)$ & 0.799 \\
\hline Aspartate aminotransferase, U/L (14-36) & $23(21,32)$ & $20(19,21)$ & 0.496 \\
\hline Total bilirubin, $\mu \mathrm{mol} / \mathrm{L}$ (3-22) & $11.1(7.2,16.1)$ & $9.4(9.4,9.4)$ & 0.444 \\
\hline Blood urea nitrogen, mmol/L (2.5-6.1) & $4.7(3.0,6.5)$ & $3.5(3.3,3.6)$ & 0.476 \\
\hline Serum creatinine, $\mu \mathrm{mol} / \mathrm{L}(46.0-92.0)$ & $47.4(42.5,69.8)$ & $58.1(54.7,61.6)$ & 0.800 \\
\hline Lactate dehydrogenase, U/L (114-240) & $246(182,313)$ & $168161,175.5)$ & 0.264 \\
\hline Procalcitonin, ng/mL $(<0.5)$ & $1.5(0.4,3.7)$ & $0.04(0.03,0.04)$ & 0.022 \\
\hline C-reactive protein, mg/L $(<8.00)$ & $68(37,126.0)$ & $3.6(2.5,4.6)$ & 0.001 \\
\hline \multicolumn{4}{|l|}{ Staging } \\
\hline Mild & 0 & 3 & 0.001 \\
\hline Common & 4 & 8 & \\
\hline Severe & 4 & 0 & \\
\hline Critical & 5 & 0 & \\
\hline \multicolumn{4}{|l|}{ Co-infection } \\
\hline Other viruses & 7 & 0 & 0.006 \\
\hline Bacteria & 11 & 3 & 0.011 \\
\hline Fungus & 9 & 0 & $<0.001$ \\
\hline \multicolumn{4}{|l|}{ Complications } \\
\hline ARDS & 6 & 0 & 0.016 \\
\hline Acute renal failure & 1 & 0 & $>0.999$ \\
\hline Sepsis & 2 & 0 & 0.482 \\
\hline
\end{tabular}


Table 2 (continued)

\begin{tabular}{lllr}
\hline & Haematological cancer, $N=13$ & Health care providers ${ }^{\mathrm{a}}, N=11$ & $P$ value \\
\hline Treatment & & & 7 \\
$\quad$ Umifenovir & 8 & 10 & 0.999 \\
Interferon & 6 & 2 & 0.037 \\
Antibiotics & 13 & 2 & 0.649 \\
Corticosteroids & 4 & 3 & 0.043 \\
Oxygen & 10 & 0 & 0.482 \\
Non-invasive ventilation & 2 & 0 & $>0.999$ \\
Mechanical ventilation & 1 & & 0.001 \\
Outcomes & & 8 & \\
Cured & 5 & 3 & 0 \\
Improved & 0 & 0 & 0 \\
Stable & 0 & 0 &
\end{tabular}

Data are $N(\%)$ unless specified.

${ }^{\mathrm{a}}$ Health care providers with COVID-19.

${ }^{\mathrm{b}}$ Lung CT scan data of subjects 1 and 5 were unavailable.

${ }^{\mathrm{c}}$ Subjects with haematological cancers and abnormal laboratory parameters were censored if seemed likely the abnormality was caused by anticancer therapies.

${ }^{\mathrm{d}}$ Follow-up date was 29 February, 2020.

acute respiratory distress syndrome (ARDS), one with acute renal disfunction and two with sepsis. No health care provider had these complications.

Persons with COVID-19 infection were treated as described [13, 24]. There were no differences between the cohorts in use of anti-virus therapy, including umifenovir and interferon, antibiotics or corticosteroids (Table 2). Subjects with haematological cancer and COVID-19 received supplemental oxygen $(N=10)$, non-invasive ventilation support $(N=2)$ and invasive ventilation $(N=1)$ compared with three controls who received supplemental oxygen.

To analyse the effect of haematological disorders on the severity and outcomes of COVID-19, we analysed data from 29 subjects with COVID-19 including the 13 subjects with haematological cancer and 11 hospitalised health care providers (Supplementary Tables 5 and 6). Four case were mild, sixteen, common, four, severe, and five, critical (Supplementary Table 3). All nine severe or critical severity cases were subjects with haematological cancer $(P=0.001)$.

Eight subjects with haematological cancers and COVID19 died compared with no controls $(P=0.001)$. Median survival of the eight subjects with haematological cancer and COVID-19 from symptom onset to death was 11 days (range, 6-29 days). Deaths were from $\operatorname{ARDS}(N=6)$, septic shock $(N=1)$ and multiple organ failure $(N=1$; Table 2 ; Supplementary Table 5). Subjects with haematological cancer and COVID-19 had a greater incidence of ARDS compared with non-COVID-19 controls (6/13 versus 0/11,
$P=0.016$ ) (Table 2; Supplementary Tables 5 and 6). And a higher incidence of ARDS was detected in non-survivors than survivors with haematological cancers $(6 / 8$ versus $0 / 5$ : $P=0.039$ ). Amongst subjects with haematological cancer and COVID-19 non-survivors had significantly higher $(P=$ 0.03 ) onset baseline D-dimer levels than survivors (Table 3 and Supplementary Table 7).

\section{Presumed cluster transmission related to intensive care unit (ICU)}

Amongst the 29 subjects with COVID-19, one had exposure to the Huanan Seafood Wholesale Market (the suspected origin site for SARS-CoV-2), seven had contact with clinically diagnosed persons with COVID-19 and one had contact with qRT-PCR-test confirmed person without COVID-19. The other 20 denied exposure to potential SARS-CoV-2 vectors. Sixteen subjects with COVID-19 were in ICUs before the COVID-19 pandemic began including eight subjects with haematological cancers and eight health care providers in the ICU as part of their work. These data compare with only 5 of 112 non-ICU subjects with haematological cancers $(P<0.001)$ and with only 13 of 289 non-ICU subjects (hospitalised subjects with haematological cancers and health care providers; $P<0.001)$. These data suggest more than one-half of COVID-19 cases we studied were ICU exposure related. In the case map an ICU nurse (patient 22) had the earliest onset time 
Fig. 1 Representative lung CT scans (transverse plane) of three subjects with COVID-19. a Ground-glass opacity in both lungs; $\mathbf{b}$ absorption of bi-lateral ground-glass opacity after treatment (patient 2); c Multiple condensation shadows in both lungs, edges were blurred; d no significant changes on image findings after treatment (patient 8); e multiple ground-glass opacity in both lungs and patchy consolidation in the right lung; and $\mathbf{f}$ diffuse condensation shadows with blurred edges; bronchiolar-inflation sign is seen in the left lung tissue area of the lesion after treatment (patient 4).

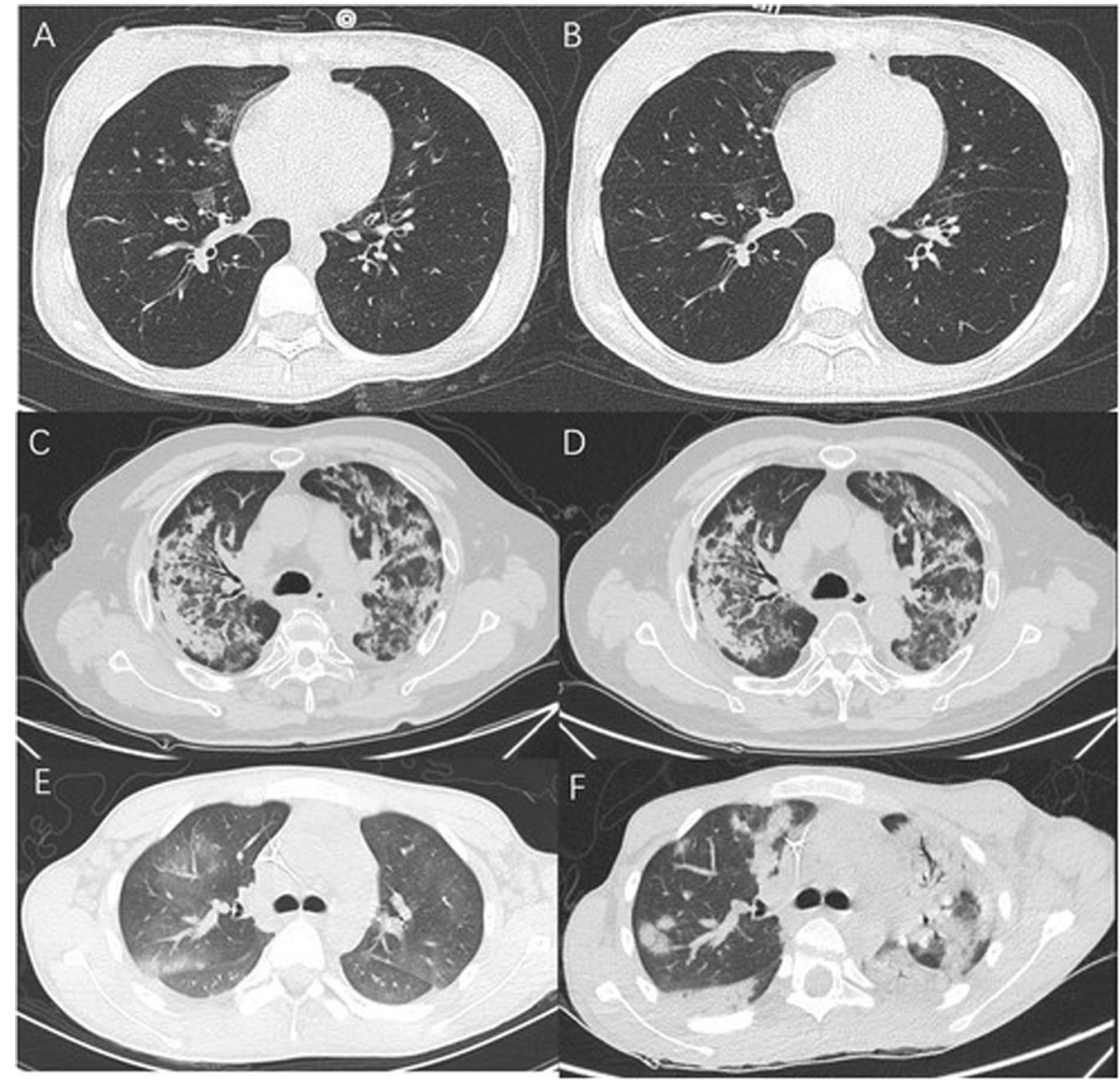

(Supplementary Fig. 1). There were correlations between and amongst more than one-half of the COVID-19 subjects we studied. We detected no more cases of COVID-19 amongst persons with a haematological cancer after the last cases with symptom onset of COVID-19 on January 31 (patient 2) and February 12 (patients 13 and 14), in Union Hospital and Wuhan Central Hospital. These data are consistent with a cluster of transmission related to ICU exposure in Union Hospital (Supplementary Tables 1-3 and Fig. 1).

\section{Discussion}

We found a $10 \%$ case rate of COVID-19 amongst 128 hospitalised persons with haematological cancer in Wuhan, much higher than reported for hospitalised persons with other cancers at another Wuhan hospital with an estimated $<1 \%$ incidence [15] but similar to the case rate of health care providers. No pre-COVID-19 co-variate could accurately predict which persons with haematological cancers were at greatest risk to develop COVID-19. We also found hospitalised subjects with haematological cancers and with COVID-19 had more severe disease and a higher case fatality rate compared with hospitalised health care providers with COVID-19. Most of our data suggest this is attributable to their haematological cancer and/or therapy thereof. One might expect persons with immune system cancers such as lymphomas and lymphoid leukaemias might be at increased risk to develop COVID-19 compared with myeloid cancer such as AML and MDS but we found no such association. The increased case fatality rate of hospitalised subjects with haematological cancers and COVID-19 seems related predominately to bacterial coinfections. This is consistent with a higher probability of decreased granulocyte concentrations because of their disease or therapy thereof.

Our study has important limitations including heterogenous subject haematological diagnoses and disease states, confounding co-variates such as therapy of haematological cancers and interval to developing COVID-19. Our diagnosis of COVID-19 was based initially on results of a screening lung CT scan but most cases were confirmed by qRT-PCR. We did not analyse infection rates with SARS-CoV-2 because tests were unavailable so are conclusions are based solely on lung CT scan findings and symptom reporting which is unreliable in subjects with haematological cancers who may have other reasons for developing fever, cough etc., especially those receiving anti-cancer therapy. Also, our start time for COVID-19 was abnormal lung CT scan or onset of symptoms 
Table 3 Clinical and laboratory co-variates and therapy of subjects with haematological cancer and COVID-19.

\begin{tabular}{|c|c|c|c|}
\hline & Survivors $N=5$ & Non-survivors $N=8$ & $P$ value \\
\hline Age, median (IQR), years & $35(26,37)$ & $39(22,54)$ & 0.665 \\
\hline Male sex & 2 & 5 & 0.826 \\
\hline \multicolumn{4}{|l|}{ Diagnosis } \\
\hline Laboratory confirmed & 5 & 8 & \multirow[t]{2}{*}{$>0.999$} \\
\hline Clinically diagnosed & 0 & 0 & \\
\hline \multicolumn{4}{|l|}{ Staging } \\
\hline Mild & 0 & 0 & \multirow[t]{4}{*}{0.194} \\
\hline Common & 3 & 1 & \\
\hline Severe & 1 & 3 & \\
\hline Critical & 1 & 4 & \\
\hline \multicolumn{4}{|l|}{ Lung CT scan } \\
\hline Patchy shadows & 1 & 1 & \multirow[t]{4}{*}{0.487} \\
\hline Ground-glass opacity & 2 & 6 & \\
\hline Air space consolidation/whiteout & 1 & 0 & \\
\hline $\mathrm{NA}^{\mathrm{a}}$ & 1 & 1 & \\
\hline \multicolumn{4}{|l|}{ Laboratory findings (normal range) } \\
\hline WBC, median (IQR), ×10E + 9/L (3.5-9.5) & $3.9(2.6,7.8)$ & $2.6(0.5,11.6)$ & 0.833 \\
\hline Neutrophils, median (IQR), ×10E + 9/L (1.8-6.3) & $2.7(1.1,6.8)$ & $0.7(0.2,6.5)$ & 0.626 \\
\hline Lymphocytes, median (IQR), ×10E + 9/L (1.1-3.2) & $0.7(0.5,0.9)$ & $0.5(0.2,0.7)$ & 0.329 \\
\hline Platelets, mean (SD), $\times 10 \mathrm{E}+9 / \mathrm{L}(125-350)$ & $107.2 \pm 86.0$ & $54.1 \pm 45.1$ & 0.168 \\
\hline $\begin{array}{l}\text { Haemoglobin, mean (SD), g/L (male, 130-175; female, } \\
115-150 \text { ) }\end{array}$ & $81.0 \pm 19.3$ & $71.9 \pm 20.6$ & 0.444 \\
\hline Prothrombin time, median (IQR), s (11.0-16.0) & $16.2(15.6,16.5)$ & $16.4(13.7,16.9)$ & $>0.999$ \\
\hline $\begin{array}{l}\text { Activated partial thromboplastin time, mean (SD), s } \\
(28.0-43.5)\end{array}$ & $44.7 \pm 10.9$ & $37.5 \pm 16.3$ & 0.408 \\
\hline D-dimer, median (IQR), mg/L $(<0.5)$ & $0.6(0.6,0.6)$ & $1.3(0.8,2.4)$ & 0.030 \\
\hline Alanine aminotransferase, mean (SD), U/L (9-52) & $18.7 \pm 10.3$ & $18.9 \pm 10.6$ & 0.0975 \\
\hline Aspartate aminotransferase, mean (SD), U/L (14-36) & $26 \pm 10$ & $24 \pm 14$ & 0.766 \\
\hline Total bilirubin, median (IQR), $\mu \mathrm{mol} / \mathrm{L}$ (3-22) & $11.1(9.6,12.9)$ & $11.7(6.0,17.2)$ & 0.943 \\
\hline Blood urea nitrogen, mean (SD), mmol/L (2.5-6.1) & $4.7 \pm 2.2$ & $5.5 \pm 3.2$ & 0.651 \\
\hline Serum creatinine, median (IQR), $\mu \mathrm{mol} / \mathrm{L}$ (46-92) & $44(41,70)$ & $56(43,68)$ & 0.622 \\
\hline Lactate dehydrogenase, median (IQR), U/L, (114-240) & $275(261,296)$ & $184.0(155,413)$ & 0.268 \\
\hline Procalcitonin, mean (SD), ng/mL $(<0.5)$ & $1.2 \pm 1.2$ & $4.0 \pm 4.2$ & 0.234 \\
\hline C-reactive protein, median $(\mathrm{IQR}), \mathrm{mg} / \mathrm{L}(<8.00)$ & $121(37,144)$ & $68(64,115)$ & $>0.999$ \\
\hline \multicolumn{4}{|l|}{ Co-infections } \\
\hline Other viruses & 3 & 4 & $>0.999$ \\
\hline Bacteria & 3 & 8 & 0.248 \\
\hline Fungi & 3 & 6 & $>0.999$ \\
\hline \multicolumn{4}{|l|}{ Complications } \\
\hline ARDS & 0 & 6 & 0.039 \\
\hline Acute renal failure & 0 & 1 & $>0.999$ \\
\hline Sepsis & 1 & 1 & $>0.999$ \\
\hline \multicolumn{4}{|l|}{ Treatment } \\
\hline Umifenovir & 3 & 5 & $>0.999$ \\
\hline Interferon & 2 & 4 & $>0.999$ \\
\hline Antibiotics & 3 & 6 & $>0.999$ \\
\hline Corticosteroids & 1 & 3 & 0.962 \\
\hline Oxygen inhalation & 3 & 7 & 0.420 \\
\hline Non-invasive ventilation & 0 & 2 & 0.671 \\
\hline Mechanical ventilation & 0 & 1 & $>0.999$ \\
\hline
\end{tabular}

${ }^{a}$ Lung CT scan data of subjects 1 and 5 were unavailable.

surveillance of which is subject to selection and recall biases. Our control cohort was health care providers rather than hospitalised persons with cancer other than haematological cancers and receiving comparably bone marrow suppressive anti-cancer therapy. Also, our data are consistent with cluster transmission related to ICU exposure.

Because we could not accurately predict which hospitalised persons with haematological cancer were at increased 
risk to develop COVID-19, we recommend special attention to SARS-CoV-2-infection and subsequent COVID-19 in hospitalised persons with haematological cancers, especially those receiving bone marrow supressing drugs and those with advanced cancers. Protective isolation should be considered, especially in persons hospitalised in the ICU.

\section{Data availability}

All data generated or analysed during this study are included in this published article and its Supplementary Information files.

Acknowledgements We thank patients, families and health care providers involved in our study. Funded by the Natural Science Foundation of China (81974009 to QL and 81974221 to ZC) and the Fundamental Research Funds for the Central Universities (2020kfyXGYJ086 to QL). RPG acknowledges support from the National Institute of Health Research Biomedical Research Centre funding scheme.

Author contributions QL, ZC and HW designed the study. QL, WH, LeC, LC, GY, YF, WC, DW, BL, XL, YM and LL collected the data. All authors had full access to the data, and were involved in data interpretation and vouch for the accuracy of the analyses. QL, WH, LeC and RPG prepared the typescript which all authors approved final approval and supported the decision to submit for publication.

\section{Compliance with ethical standards}

Conflict of interest The authors declare that they have no conflict of interest.

Publisher's note Springer Nature remains neutral with regard to jurisdictional claims in published maps and institutional affiliations.

\section{References}

1. Chan JF-W, Kok K-H, Zhu Z, Chu H, To KK-W, Yuan S, et al. Genomic characterization of the 2019 novel human-pathogenic coronavirus isolated from a patient with atypical pneumonia after visiting Wuhan. Emerg Microbes Infect. 2020;9:221-36.

2. Lu R, Zhao X, Li J, Niu P, Yang B, Wu H, et al. Genomic characterisation and epidemiology of 2019 novel coronavirus: implications for virus origins and receptor binding. Lancet. 2020;395:565-74.

3. Gorbalenya AE, Baker SC, Baric RS, de Groot RJ, Drosten C, Gulyaeva AA, et al. The species severe acute respiratory syndrome-related coronavirus: classifying 2019-nCoV and naming it SARS-CoV-2. Nat Microbiol. 2020;5:536-44.

4. Wu Z, McGoogan JM. Characteristics of and important lessons from the coronavirus disease 2019 (COVID-19) outbreak in China: summary of a report of 72,314 cases from the Chinese Center for Disease Control and Prevention. JAMA. 2020. https://doi.org/10.1001/jama.2020.2648.

5. Guan W-j, Ni Z-y, Hu Y, Liang W-h, Ou C-q, He J-x, et al. Clinical characteristics of coronavirus disease 2019 in China. $\mathrm{N}$ Engl J Med. 2020. https://doi.org/10.1056/NEJMoa2002032.

6. Huang C, Wang Y, Li X, Ren L, Zhao J, Hu Y, et al. Clinical features of patients infected with 2019 novel coronavirus in Wuhan, China. Lancet. 2020;395:497-506.
7. Wang C, Horby PW, Hayden FG, Gao GF. A novel coronavirus outbreak of global health concern. Lancet. 2020;395:470-3.

8. Gates B. Responding to Covid-19-a once-in-a-century pandemic? N Engl J Med. 2020;382:692-4.

9. Wang D, Hu B, Hu C, Zhu F, Liu X, Zhang J, et al. Clinical characteristics of 138 hospitalized patients with 2019 novel coronavirus-infected pneumonia in Wuhan, China. JAMA. 2020; 323:1061-9.

10. Chen N, Zhou M, Dong X, Qu J, Gong F, Han Y, et al. Epidemiological and clinical characteristics of 99 cases of 2019 novel coronavirus pneumonia in Wuhan, China: a descriptive study. Lancet. 2020;395:507-13.

11. Zhu N, Zhang D, Wang W, Li X, Yang B, Song J, et al. A novel coronavirus from patients with pneumonia in China, 2019. N Engl J Med. 2020;382:727-33.

12. Shi H, Han X, Jiang N, Cao Y, Alwalid O, Gu J, et al. Radiological findings from 81 patients with COVID-19 pneumonia in Wuhan, China: a descriptive study. Lancet Infec Dis. 2020. https://doi.org/10.1016/S1473-3099(20)30086-4.

13. Yang X, Yu Y, Xu J, Shu H, Xia Ja, Liu H, et al. Clinical course and outcomes of critically ill patients with SARS-CoV-2 pneumonia in Wuhan, China: a single-centered, retrospective, observational study. Lancet Resp Med. https://doi.org/10.1016/S22132600(20)30079-5.

14. Liang W, Guan W, Chen R, Wang W, Li J, Xu K, et al. Cancer patients in SARS-CoV-2 infection: a nationwide analysis in China. Lancet Oncol. 2020;21:335-7.

15. Yu J, Ouyang W, Chua MLK, Xie C. SARS-CoV-2 transmission in patients with cancer at a tertiary care hospital in Wuhan, China. JAMA Oncol. 2020. https://doi.org/10.1001/jamaoncol.2020.0980.

16. Kutikov A, Weinberg DS, Edelman MJ, Horwitz EM, Uzzo RG, Fisher RI. A war on two fronts: cancer care in the time of COVID19. Ann Intern Med. 2020. https://doi.org/10.7326/m20-1133.

17. The Lancet O. COVID-19: global consequences for oncology. Lancet Oncol. 2020;21:467.

18. Xia Y, Jin R, Zhao J, Li W, Shen H. Risk of COVID-19 for patients with cancer. Lancet Oncol. 2020;21:e180.

19. Ludwig H, Delforge M, Facon T, Einsele H, Gay F, Moreau P, et al. Prevention and management of adverse events of novel agents in multiple myeloma: a consensus of the European Myeloma Network. Leukemia. 2018;32:1542-60.

20. Maschmeyer G, De Greef J, Mellinghoff SC, Nosari A, ThiebautBertrand A, Bergeron A, et al. Infections associated with immunotherapeutic and molecular targeted agents in hematology and oncology. A position paper by the European Conference on Infections in Leukemia (ECIL). Leukemia. 2019;33:844-62.

21. Taplitz RA, Kennedy EB, Bow EJ, Crews J, Gleason C, Hawley DK, et al. Antimicrobial prophylaxis for adult patients with cancer-related immunosuppression: ASCO and IDSA clinical practice guideline update. J Clin Oncol. 2018;36:3043-54.

22. National Health Commission of China. The novel coronavirus pneumonia diagnosis and treatment program, 5th version. China: 2020; http://www.nhc.gov.cn/yzygj/s7653p/202002/3b09b894a c9b4204a79db5b8912d4440.shtml. Accessed 26 Feb.

23. WHO. Clinical management of severe acute respiratory infection when novel coronavirus ( $\mathrm{nCoV})$ infection is suspected: interim guidance. Switzerland: WHO; 2020. https://www.who.int/publica tions-detail/clinical-management-of-severe-acute-respiratoryinfection-when-novel-coronavirus-(ncov)-infection-is-suspected. Accessed 25 Feb.

24. Zhang J, Zhou L, Yang Y, Peng W, Wang W, Chen X. Therapeutic and triage strategies for 2019 novel coronavirus disease in fever clinics. Lancet Resp Med. 2020;8:e11-2. 